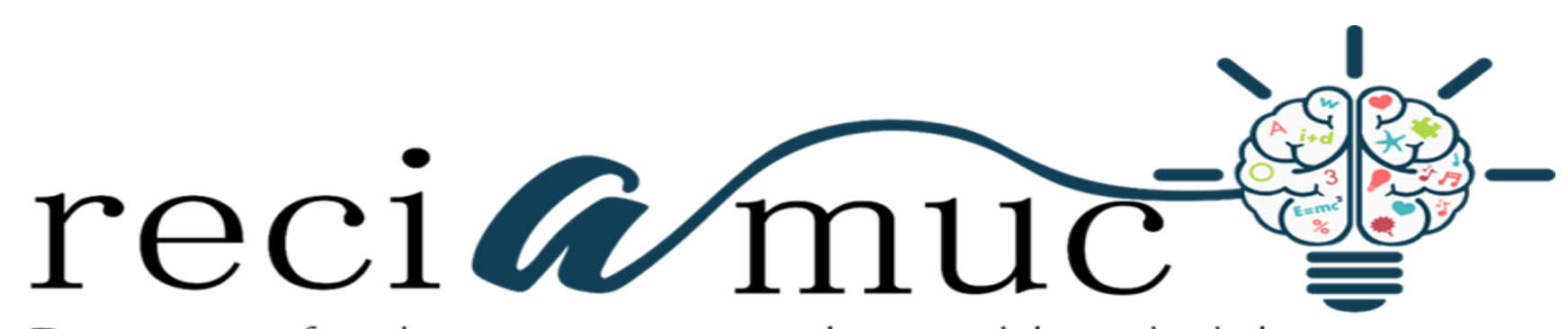

Revista cientifica de investigación actualización del mundo de las ciencias

Stefany Gianella Campuzano Lupera ${ }^{\text {a; }}$ Shirley Natali Lema Sarmiento ${ }^{\text {b }}$ Elvia María Córdova Cedeño ${ }^{c}$; Luis Alberto Criollo Cartuche ${ }^{\mathrm{d}}$

Tratamiento de las infecciones del tracto urinario en embarazo

Treatment of urinary tract infections in pregnancy

Revista Científica de Investigación actualización del mundo de las Ciencias. Vol. 3 núm., 2, abril, ISSN: 2588-0748, 2018, pp. 439-458

DOI: $10.26820 / \mathrm{reciamuc/3.(2).abril.2019.439-458}$

URL: http://reciamuc.com/index.php/RECIAMUC/article/view/348

Código UNESCO: 3205 Medicina Interna

Tipo de Investigación: Artículo de Revisión

(C) RECIAMUC; Editorial Saberes del Conocimiento, 2019

Recibido: 15/01/2019

Aceptado: 07/02/2019

Publicado: 01/04/2019

Correspondencia: stefany.campuzano@gmail.com
a. Médico; Investigador Independiente; Guayaquil, Ecuador; stefany.campuzano@ gmail.com
b. Médico; Investigador Independiente; Guayaquil, Ecuador; shirleylemast@ gmail.com
c. Médico; Investigador Independiente; Guayaquil, Ecuador; eccmd.84@ hotmail.com
d. Médico; Investigador Independiente; Guayaquil, Ecuador; luiscriollo182@gmail.com 


\section{Tratamiento de las infecciones del tracto urinario en embarazo}

Vol. 3, núm. 2., (2019)

Stefany Gianella Campuzano Lupera; Shirley Natali Lema Sarmiento; Elvia María Córdova Cedeño; Luis Alberto Criollo Cartuche

\section{RESUMEN}

Las infecciones urinarias son las infecciones bacterianas más comunes durante el embarazo. Se caracterizan por la presencia de bacterias significativas en cualquier parte del tracto urinario. La pielonefritis es la infección bacteriana grave más común que puede conducir a complicaciones maternas y perinatales, incluyendo parto prematuro, bebés con bajo peso al nacer, mortalidad fetal, preeclampsia, hipertensión inducida por el embarazo, anemia, trombocitopenia e insuficiencia renal transitoria. Las enterobacterias representan el $90 \%$ de las infecciones urinarias. Los antibióticos comunes utilizados son nitrofurantoína, cefazolina, cefalexina, ceftriaxona y gentamicina. El tratamiento terapéutico de las infecciones urinarias en el embarazo requiere una evaluación diagnóstica adecuada y una comprensión profunda de los agentes antimicrobianos para optimizar el resultado materno, garantizar la seguridad del feto y prevenir complicaciones que conducen a una morbilidad y mortalidad significativas tanto en el feto como en la madre. El propósito de esta guía es proporcionar recomendaciones multidisciplinares para el tratamiento de mujeres con bacteriuria asintomática e infecciones del tracto urinario en el embarazo. Estas directrices están destinadas a profesionales de la salud, en particular los de la formación, que están trabajando en los servicios de obstetricia y ginecología. Están diseñados para guiar el juicio clínico, pero no sustituirla. En casos individuales, un profesional de la salud puede, después de una cuidadosa consideración, decida no seguir una pauta si se considera que es en el mejor interés de la mujer.

Palabras claves: Bacteriuria; Cistitis; Gestación; Embarazo; Pielonefritis; Infección del tracto urinario. 


\title{
Tratamiento de las infecciones del tracto urinario en embarazo
}

Vol. 3, núm. 2., (2019)

Stefany Gianella Campuzano Lupera; Shirley Natali Lema Sarmiento; Elvia María Córdova Cedeño; Luis Alberto Criollo Cartuche

\begin{abstract}
Urinary infections are the most common bacterial infections during pregnancy. They are characterized by the presence of significant bacteria in any part of the urinary tract. Pyelonephritis is the most common serious bacterial infection that can lead to maternal and perinatal complications, including premature delivery, low birth weight babies, fetal mortality, preeclampsia, pregnancy-induced hypertension, anemia, thrombocytopenia and transient renal failure. Enterobacteria represent $90 \%$ of urinary infections. The common antibiotics used are nitrofurantoin, cefazolin, cephalexin, ceftriaxone and gentamicin. The therapeutic treatment of urinary tract infections in pregnancy requires an adequate diagnostic evaluation and a deep understanding of antimicrobial agents to optimize the maternal outcome, guarantee the safety of the fetus and prevent complications that lead to significant morbidity and mortality in both the fetus and in the mother. The purpose of this guide is to provide multidisciplinary recommendations for the treatment of women with asymptomatic bacteriuria and urinary tract infections in pregnancy. These guidelines are intended for health professionals, particularly those for training, who are working in obstetrics and gynecology services. They are designed to guide clinical judgment, but not replace it. In individual cases, a health professional may, after careful consideration, decide not to follow a pattern if it is considered to be in the best interest of the woman.
\end{abstract}

Key words: Bacteriuria; Cystitis; Pregnancy; Pregnancy; Pyelonephritis; Urinary tract infection. 


\section{Tratamiento de las infecciones del tracto urinario en embarazo}

Vol. 3, núm. 2., (2019)

Stefany Gianella Campuzano Lupera; Shirley Natali Lema Sarmiento; Elvia María Córdova Cedeño; Luis Alberto Criollo Cartuche

\section{Introducción.}

Infecciones del tracto urinario (ITU) son una de las complicaciones más frecuentes durante el embarazo (Overturf, y otros 1992). Tradicionalmente UTI se clasifica como que afecta al tracto urinario inferior (cistitis aguda) o el tracto urinario superior (pielonefritis aguda). Un factor de predisposición o precursor de UTI es la bacteriuria.

La bacteriuria asintomática se define como la presencia de un cultivo de orina positivo en una persona asintomática y se produce en 2 a 7 por ciento de todos los embarazos (Patterson y Andriole 1997). Las tasas de bacteriuria asintomática en la población embarazadas y no embarazadas son similares, sin embargo, bacteriuria durante el embarazo tiene una mayor tendencia a progresar a infección ascendente que en la mujer no embarazada, esto es porque el embarazo se asocia con un rápido aumento en los niveles de progesterona lo que conduce a la dilatación ureteral y estasis urinaria, que aumenta el riesgo de bacteriuria. La presión mecánica desde el útero grávido y los cambios fisiológicos que ocurren en el embarazo aumentan aún más el riesgo de bacteriuria asintomática y en la infección por su vez ascendente (Perera 2009).

La bacteriuria asintomática se asocia con un mayor riesgo de resultados adversos en el feto. En particular, un aumento del riesgo de parto prematuro y un aumento del riesgo de tener un bebé con bajo peso al nacer. Además, los estudios también han demostrado que el tratamiento de la bacteriuria asintomática durante el embarazo reduce la incidencia de estas complicaciones. El rápido reconocimiento y tratamiento de la bacteriuria, por lo tanto, deben limitar el riesgo de progresión a la infección ascendente y el riesgo de estos los resultados maternos y fetales adversos. 


\section{Tratamiento de las infecciones del tracto urinario en embarazo}

Vol. 3, núm. 2., (2019)

Stefany Gianella Campuzano Lupera; Shirley Natali Lema Sarmiento; Elvia María Córdova Cedeño; Luis Alberto Criollo Cartuche

\section{Metodología.}

Para el desarrollo de este proceso investigativo, se plantea como metodología la encaminada hacia una orientación científica particular que se encuentra determinada por la necesidad de indagar en forma precisa y coherente una situación, en tal sentido (Davila, 2015) define la metodología "como aquellos pasos previos que son seleccionados por el investigador para lograr resultados favorables que le ayuden a plantear nuevas ideas”. (p.66)

Lo citado por el autor, lleva a entender que el desarrollo de la acción investigativa busca simplemente coordinar acciones enmarcadas en una revisión bibliográfica con el fin de complementar ideas previas relacionadas al manejo de las infecciones del tracto urinario durante el embarazo a través de una revisión de literatura, para así finalmente elaborar un cuerpo de consideraciones generales que ayuden a ampliar el interés propuesto.

\section{Tipo de Investigación.}

Dentro de toda práctica investigativa, se precisan acciones de carácter metodológico mediante las cuales, se logra conocer y proyectar los eventos posibles que la determinan, así como las características que hacen del acto científico un proceso interactivo ajustado a una realidad posible de ser interpretada. En este sentido, se puede decir, que la presente investigación corresponde al tipo documental, definido por Castro (2016), "se ocupa del estudio de problemas planteados a nivel teórico, la información requerida para abordarlos se encuentra básicamente en materiales impresos, audiovisuales y /o electrónicos”. (p.41). 


\section{Tratamiento de las infecciones del tracto urinario en embarazo}

Vol. 3, núm. 2., (2019)

Stefany Gianella Campuzano Lupera; Shirley Natali Lema Sarmiento; Elvia María Córdova Cedeño; Luis Alberto Criollo Cartuche

En consideración a esta definición, la orientación metodológica permitió la oportunidad de cumplir con una serie de actividades inherentes a la revisión y lectura de diversos documentos donde se encontraron ideas explicitas relacionadas con los tópicos encargados de identificar a cada característica insertada en el estudio. Por lo tanto, se realizaron continuas interpretaciones con el claro propósito de revisar aquellas apreciaciones o investigaciones propuestas por diferentes investigadores relacionadas con el tema de interés, para luego dar la respectiva argumentación a los planteamientos, en función a las necesidades encontradas en la indagación.

\section{Fuentes Documentales.}

El análisis correspondiente a las características que predomina en el tema seleccionado, llevan a incluir diferentes fuentes documentales encargadas de darle el respectivo apoyo y en ese sentido cumplir con la valoración de los hechos a fin de generar nuevos criterios que sirven de referencia a otros procesos investigativos. Para (CASTRO, 2016) las fuentes documentales incorporadas en la investigación documental o bibliográfica, "representa la suma de materiales sistemáticos que son revisados en forma rigurosa y profunda para llegar a un análisis del fenómeno". (p.41). Por lo tanto, se procedió a cumplir con la realización de una lectura previa determinada para encontrar aquellos aspectos estrechamente vinculados con el tema, con el fin de explicar mediante un desarrollo las respectivas apreciaciones generales de importancia.

\section{Técnicas para la Recolección de la Información.}

La conducción de la investigación para ser realizada en función a las particularidades que determinan a los estudios documentales, tiene como fin el desarrollo de un conjunto de acciones 


\section{Tratamiento de las infecciones del tracto urinario en embarazo}

Vol. 3, núm. 2., (2019)

Stefany Gianella Campuzano Lupera; Shirley Natali Lema Sarmiento; Elvia María Córdova Cedeño; Luis Alberto Criollo Cartuche

encargadas de llevar a la selección de técnicas estrechamente vinculadas con las características del estudio. En tal sentido, (Bolívar, 2015), refiere, que es "una técnica particular para aportar ayuda a los procedimientos de selección de las ideas primarias y secundarias”. (p. 71).

Por ello, se procedió a la utilización del subrayado, resúmenes, fichaje, como parte básica para la revisión y selección de los documentos que presentan el contenido teórico. Es decir, que mediante la aplicación de estas técnicas se pudo llegar a recoger informaciones en cuanto a la revisión bibliográfica de los diversos elementos encargados de orientar el proceso de investigación. Tal como lo expresa, (Bolívar, 2015) "las técnicas documentales proporcionan las herramientas esenciales y determinantes para responder a los objetivos formulados y llegar a resultados efectivos" (p. 58). Es decir, para responder con eficiencia a las necesidades investigativas, se introdujeron como técnica de recolección el método inductivo, que hizo posible llevar a cabo una valoración de los hechos de forma particular para llegar a la explicación desde una visión general.

Asimismo, se emplearon las técnicas de análisis de información para la realización de la investigación que fue ejecutada bajo la dinámica de aplicar diversos elementos encargados de determinar el camino a recorrer por el estudio, según, (Bolívar, 2015) las técnicas de procesamiento de datos en los estudios documentales "son las encargadas de ofrecer al investigador la visión o pasos que debe cumplir durante su ejercicio, cada una de ellas debe estar en correspondencia con el nivel a emplear" (p. 123). Esto indica, que para llevar a cabo el procesamiento de los datos obtenidos una vez aplicado las técnicas seleccionadas, tales como: fichas de resumen, textual, registros descriptivos entre otros, los mismos se deben ajustar al nivel que ha sido seleccionado. 


\section{Tratamiento de las infecciones del tracto urinario en embarazo}

Vol. 3, núm. 2., (2019)

Stefany Gianella Campuzano Lupera; Shirley Natali Lema Sarmiento; Elvia María Córdova Cedeño; Luis Alberto Criollo Cartuche

\section{Resultados}

\begin{tabular}{|l|l|}
\hline BD & Dos veces al día \\
\hline CI & Intervalo de confianza \\
\hline IV & Intravenoso \\
\hline sobredosis & \\
\hline correos & Una vez al día \\
\hline QDS & tomar por vía oral \\
\hline RR & Cuatro veces al día \\
\hline infección del tracto urinario & Radio de riesgo \\
\hline
\end{tabular}

Los signos y síntomas

La bacteriuria asintomática es una infección ascendente que se puede presentar en el embarazo con dolor abdominal bajo, frecuencia, disuria, hematuria, vómitos o pirexia y en los casos en pielonefritis ha ocurrido signos de infección sistémica puede estar presente además de dolor en el flanco (Tabla 1). 


\section{Tratamiento de las infecciones del tracto urinario en embarazo}

Vol. 3, núm. 2., (2019)

Stefany Gianella Campuzano Lupera; Shirley Natali Lema Sarmiento; Elvia María Córdova Cedeño; Luis Alberto Criollo Cartuche

En la pielonefritis severa existe un riesgo importante de progresión a la sepsis sistémica y en algunos casos de insuficiencia respiratoria aguda. La pielonefritis no tratada puede conducir a la formación de abscesos y supuración (es decir, la descarga de pus) (Cunningham y Lucas 1994).

En el embarazo la frecuencia urinaria es común como la vejiga y el útero grávido compiten por el espacio en la pelvis. Desafortunadamente, los cambios sintomatología UTI en el embarazo y la disuria no pueden estar presentes. En algunos casos los vómitos pueden recurrir o aumentar en frecuencia y puede ser la única indicación de que la infección está presente. Algoritmos de diagnósticos clínicos actuales para la detección de la IU cuando se aplica a la mujer embarazada tienden a ser decepcionantes ya que tienen baja especificidad y valores predictivos positivos.

Tabla 1: Signos y síntomas de infecciones del tracto urinario clínicas.

\begin{tabular}{|c|c|c|}
\hline $\begin{array}{c}\text { Asintomática } \\
\text { bacteriuria }\end{array}$ & IU baja / cistitis & $\begin{array}{c}\text { Infección del tracto } \\
\text { urinario superior / } \\
\text { Pielonefritis }\end{array}$ \\
\hline \multirow[t]{4}{*}{$\begin{array}{c}\text { No hay signos clínicos o } \\
\text { síntomas }\end{array}$} & Frecuencia & Pirexia \\
\hline & disuria & Dolor lumbar \\
\hline & fiebre de bajo grado & disuria \\
\hline & dolor suprapúbico & rigores \\
\hline
\end{tabular}




\section{Tratamiento de las infecciones del tracto urinario en embarazo}

Vol. 3, núm. 2., (2019)

Stefany Gianella Campuzano Lupera; Shirley Natali Lema Sarmiento; Elvia María Córdova Cedeño; Luis Alberto Criollo Cartuche

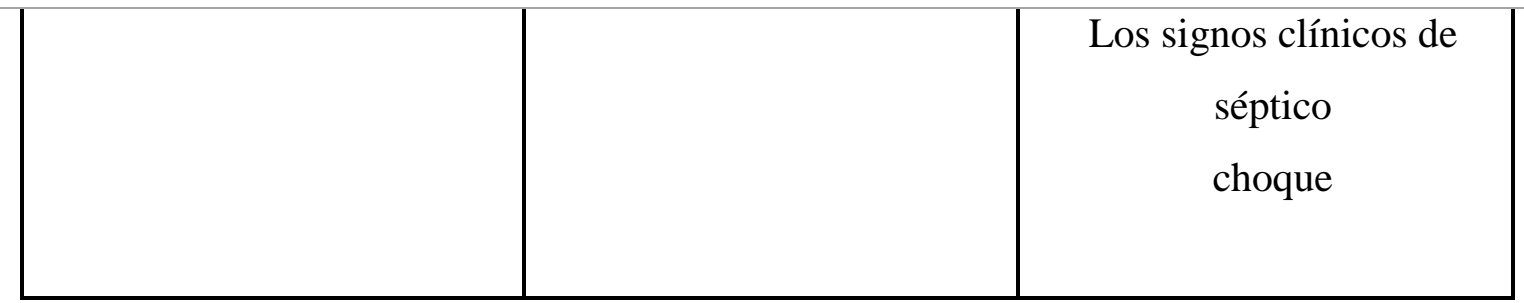

\section{Diagnóstico de laboratorio}

La detección de la bacteriuria asintomática debe realizarse idealmente a las 12 a 16 semanas de gestación en todas las mujeres. El diagnóstico de la bacteriuria asintomática se hace después del aislamiento de un crecimiento significativo de una especie bacteriana en una muestra "limpia" de captura de orina. Una toma limpia de espécimen implica la recogida de una muestra de medio de la corriente de orina después de la limpieza del perineo con el fin de minimizar la contaminación por la flora de la piel.

La microscopía y cultivo de orina siguen siendo el estándar de oro en la detección de la bacteriuria asintomática. Sin embargo, puede tardar 48 horas para obtener un resultado. Pruebas de selección rápida se han desarrollado. Los análisis de varilla de orina reactivo de nivel proporcionan una prueba barata, rápida y fácil para la bacteriuria, tasas de sensibilidad, sin embargo, los estudios han reportado asintomáticos entre 50 y 90\% con una especificidad del 83 al 94\% (Etherington y James 1993). Otra comparación de estudio de centrifugación y tinción de Gram de orina, análisis de orina y reactivo de ensayo de tira en aislamiento y en combinación mostraron altas tasas de falsos negativos con análisis de orina $(19,4 \%)$ y las pruebas de tira de reactivo $(52,8 \%)$ y baja especificidad del frotis teñido con Gram centrifugó $(7,7 \%)$ en comparación con la reportada sensibilidad 80\% con cultivo de orina (Pastore, Savitz y Thorp 1999). 


\section{Tratamiento de las infecciones del tracto urinario en embarazo}

Vol. 3, núm. 2., (2019)

Stefany Gianella Campuzano Lupera; Shirley Natali Lema Sarmiento; Elvia María Córdova Cedeño; Luis Alberto Criollo Cartuche

Investigaciones radiológicas

Imágenes del tracto renal puede estar justificada donde la pielonefritis sea recurrente o es lento para responder al tratamiento. Se trata de identificar anomalías renales o cálculos. También se debe utilizar en los casos en que se sospecha de un absceso renal, hematuria persiste o se sospecha de malignidad. Ultrasonido (US) es la investigación radiológica primaria de elección para la evaluación del tracto renal en el embarazo.

Las medidas no farmacológicas y profilaxis antimicrobiana

Pielonefritis puede volver a ocurrir durante el embarazo. Un estudio demostró una tasa de recurrencia de aproximadamente $20 \%$ durante el embarazo o el período postnatal. Una revisión Cochrane en 2008 de diez estudios mostraron que el jugo de arándano qué disminuye el número de IU durante doce meses, pero no existen datos sobre el momento óptimo y la cantidad de jugo de arándano que debe ser consumida para prevenir la infección (Jepson 2008).

El enfoque en la prevención de la recurrencia se ha centrado en medidas farmacológicas y no farmacológicas. Una revisión Cochrane mostró que dosis oral de nitrofurantoína baja en combinación con el aumento de revisión clínica y la vigilancia no ha demostrado superioridad sobre aumento de la vigilancia sólo en la prevención de la recurrencia de la infección del tracto urinario. Además, no se observó ninguna diferencia en la incidencia de parto prematuro en este grupo. El uso de nitrofurantoína y una política de estrecha vigilancia se ha traducido en una reducción del tipo de la bacteriuria asintomática y puso de relieve la necesidad de un gran ensayo 


\section{Tratamiento de las infecciones del tracto urinario en embarazo}

Vol. 3, núm. 2., (2019)

Stefany Gianella Campuzano Lupera; Shirley Natali Lema Sarmiento; Elvia María Córdova Cedeño; Luis Alberto Criollo Cartuche

controlado aleatorio para determinar qué medidas, en su caso, pueden reducir el riesgo de infecciones urinarias recurrentes.

\section{La gestión clínica}

La bacteriuria asintomática

- El tratamiento antibiótico de la bacteriuria asintomática en el embarazo reduce el riesgo de infección del tracto urinario, parto prematuro y bebés de bajo peso de nacimiento.

- Todas las mujeres deben ser examinados para la bacteriuria asintomática en la primera visita prenatal.

- Tratar a mujeres con un cultivo de orina positivo para bacteriuria detectados durante el embarazo con un antibiótico apropiado para las bacterias aisladas y el trimestre del embarazo.

- Consulte las normativas locales y nacionales para la elección del antibiótico en el embarazo

- Un curso de siete días de tratamiento es normalmente suficiente.

- No prescribir trimetoprima para las mujeres embarazadas con deficiencia de folato establecido o las mujeres que toman antagonistas de folato.

La bacteriuria sintomática

- Cultivo de orina es la prueba de elección en la bacteriuria sintomática.

- Tratar bacteriuria sintomática con un antibiótico de acuerdo con la guía local.

- Permite tomar una sola muestra para cultivo de orina antes de iniciar el tratamiento empírico. 


\section{Tratamiento de las infecciones del tracto urinario en embarazo}

Vol. 3, núm. 2., (2019)

Stefany Gianella Campuzano Lupera; Shirley Natali Lema Sarmiento; Elvia María Córdova Cedeño; Luis Alberto Criollo Cartuche

- Las mujeres con bacteriuria sintomáticos con signos sistémicos de infección deben ser admitidos a antibióticos por vía intravenosa en espera del resultado de los cultivos de sangre y el cultivo de orina

- En los casos en que una mejoría clínica no se produce con 24 horas de instigar el tratamiento o cuando existen comorbilidades adicionales de alto consejo médico y la microbiología se buscaran nuevos

- Imágenes renales se debe considerar si la patología renal se sospecha o en los casos que se repiten

\section{Tratamiento farmacológico}

- Principios generales de uso de antimicrobianos

La elección del antimicrobiano adecuado es una parte esencial de la gestión de pacientes embarazadas con infecciones del tracto urinario. No sólo es importante elegir el medicamento correcto, sino que también debe considerarse la posibilidad de seleccionar la duración de la dosis y el tratamiento correcto. Al tratar eficazmente las infecciones del tracto urinario se espera para reducir el riesgo de sepsis materna, pielonefritis, trabajo de parto prematuro y los resultados también adversos para el feto. También se debe considerar la posibilidad de teratogenicidad potencial de la hora de elegir un antimicrobiano. Esto puede ser más difícil en el entorno de alergia a la penicilina, pero los riesgos y beneficios debe ser explicado al paciente. 


\section{Tratamiento de las infecciones del tracto urinario en embarazo}

Vol. 3, núm. 2., (2019)

Stefany Gianella Campuzano Lupera; Shirley Natali Lema Sarmiento; Elvia María Córdova Cedeño; Luis Alberto Criollo Cartuche

\section{- Bacteriuria asintomática}

La principal evidencia para el tratamiento de la bacteriuria asintomática durante el embarazo para prevenir resultados adversos maternos y fetales surge de una revisión Cochrane en 2000 que mostró que cuando el tratamiento antimicrobiano se comparó con placebo o ningún tratamiento era eficaz en (Smaill 2000):

a) Eliminación de bacteriuria asintomática (razón de riesgo (RR) 0,25, 95\% intervalo de confianza (CI) 0,14-0,48)

b) La reducción del riesgo de pielonefritis ( $\mathrm{RR}$ 0,23, IC del 95\%: 0,13 a 0,41)

c) La reducción de la incidencia de bebés con bajo peso de nacimiento (RR 0,66, IC del 95\%: $0,49$ a 0,89$)$

Una revisión Cochrane de diferentes regímenes de tratamiento antimicrobiano de la bacteriuria asintomática en el embarazo no se encontró diferencias significativas en términos de resultados entre varios regímenes y sugirió que las cuestiones tales como los resultados locales de susceptibilidad, costo y los efectos secundarios deben ser tomados en cuenta (Guinto, y otros 2010). Una tercera revisión Cochrane que se centró en la duración del tratamiento antimicrobiano en la bacteriuria asintomática en el embarazo revisó 13 estudios, que incluyeron más de 1.622 mujeres. Se encontró que un régimen de un día es significativamente menos eficaz que un régimen de siete días, y que la práctica actual debe ser para tratar pacientes durante siete días (Widmer, y otros 2011). 


\section{Tratamiento de las infecciones del tracto urinario en embarazo}

Vol. 3, núm. 2., (2019)

Stefany Gianella Campuzano Lupera; Shirley Natali Lema Sarmiento; Elvia María Córdova Cedeño; Luis Alberto Criollo Cartuche

Se han realizado varios ensayos que han demostrado que los siguientes regímenes son eficaces en el tratamiento de la bacteriuria asintomática en el embarazo.
a) TDS coamoxiclav $625 \mathrm{mg}$ durante 5 días o durante 7 días
b) Cefuroxima axetilo $250 \mathrm{mg}$ BD o $500 \mathrm{mg}$ BD durante 5 días
c) 500mg cefaclor durante 5-7 días para las mujeres con hipersensibilidad a la penicilina leve
d) Dosis stat fosfomicina $3 \mathrm{~g}$
e) Nitrofurantoin $100 \mathrm{mg}$ BD PO durante 7 días

Al elegir un régimen antimicrobiano es importante tener en cuenta cuestiones de teratogenicidad y la absorción. Las recomendaciones se incluyen en la Tabla 1.

IU baja (cistitis) en el embarazo

Una revisión Cochrane de tratamiento de las infecciones urinarias sintomáticas en el embarazo encontró que no había pruebas suficientes para recomendar cualquier régimen de fármaco específico, y que todos los regímenes estudiados demostraron ser muy eficaces (Vázquez y Abalos 2011)

Hay pocos estudios disponibles sobre el tratamiento de las infecciones urinarias bajas sintomáticas durante el embarazo y el único estudio encontrado en nuestra búsqueda había dos regímenes que se mostraron como equivalentes:
a) La fosfomicina $3 \mathrm{~g}$ dosis stat $\mathrm{PO}$
b) Ceftibuten PO $400 \mathrm{mg}$ durante 3 días 


\section{Tratamiento de las infecciones del tracto urinario en embarazo}

Vol. 3, núm. 2., (2019)

Stefany Gianella Campuzano Lupera; Shirley Natali Lema Sarmiento; Elvia María Córdova Cedeño; Luis Alberto Criollo Cartuche

Para el tratamiento de las infecciones urinarias sintomáticas durante el embarazo se recomienda para el tratamiento de siete días, excepto en el caso en que se utiliza la fosfomicina. Un nuevo urocultivo debe enviarse una semana después del tratamiento antimicrobiano está terminado para asegurar que la bacteriuria ha despejado.

\section{Alta UTI (pielonefritis) en el embarazo}

Existen varias directrices internacionales. Un pequeño estudio de 67 mujeres comparó el tratamiento IV solo hasta que el paciente era de 48 horas afebril al tratamiento IV con PO antimicrobianos después de terminar un total de 10 días de tratamiento. Se encontró que en las mujeres sin antimicrobianos orales adicionales tuvo la oportunidad 12,9\% de la readmisión de pielonefritis en el período de 2 semanas después de la infección, en comparación con una tasa de readmisión 5,6\% en las mujeres que recibieron antibióticos orales para terminar el curso de 10 días de tratamiento.

Hay datos de ensayos limitados disponibles para el tratamiento de la pielonefritis prenatal, sin embargo, los regímenes que se han utilizado se muestran a continuación.

a) Ceftriaxona $1 \mathrm{~g}$ al día hasta 48 horas QDS cefalexina $500 \mathrm{mg}$ afebril entonces orales durante 10 días

b) La cefazolina $1 \mathrm{~g}$ o $2 \mathrm{~g}$ TDS hasta 48 horas QDS cefalexina $500 \mathrm{mg}$ afebril entonces orales durante 10 días

c) Ceftriaxona $1 \mathrm{~g}$ IM para 2 dosis orales entonces QDS cefalexina $500 \mathrm{mg}$, por 10 días 


\section{Tratamiento de las infecciones del tracto urinario en embarazo}

Vol. 3, núm. 2., (2019)

Stefany Gianella Campuzano Lupera; Shirley Natali Lema Sarmiento; Elvia María Córdova Cedeño; Luis Alberto Criollo Cartuche

La preocupación por la gestión de pacientes en las etapas iniciales es que la mujer puede progresar en el trabajo y deben ser admitidos durante al menos 48 horas si se sospecha de pielonefritis. Una preocupación con el uso de dosis de $1 \mathrm{~g}$ de ceftriaxona es que cuando las mujeres están en el tercer trimestre pueden estar bajo dosificados y dosis mayores (hasta 2 g una vez al día) deben ser considerados. También hay incertidumbre en torno a la frecuencia de dosificación de gentamicina en el embarazo.

Profilaxis antimicrobiana para las infecciones del tracto urinarioen el embarazo

La profilaxis antimicrobiana debe considerarse también en las mujeres en los siguientes grupos

- Pielonefritis en el embarazo

- Conocido anormalidad estructural renal que conduce a estasis urinaria

- Infecciones recurrentes del tracto urinario

El uso de la profilaxis antimicrobiana para las infecciones urinarias recurrentes o siguiente pielonefritis es un área difícil y no hay indicaciones basadas en la evidencia claro en la actualidad. Nitrofurantoin se puede utilizar para la profilaxis, pero se debe evitar a corto plazo o cuando el parto es inminente, debido al riesgo de hemólisis neonatal. El uso de antimicrobianos de amplio espectro tales como amoxicilina-ácido clavulánico y cefalexina debe hacerse después de la evaluación de los beneficios de la profilaxis contra el riesgo de generar resistencia a los antimicrobianos en el paciente que lleva a las opciones de tratamiento más limitadas en una etapa 


\section{Tratamiento de las infecciones del tracto urinario en embarazo}

Vol. 3, núm. 2., (2019)

Stefany Gianella Campuzano Lupera; Shirley Natali Lema Sarmiento; Elvia María Córdova Cedeño; Luis Alberto Criollo Cartuche

posterior. Se necesita más investigación en esta área y se ha destacado en la sección de preguntas de investigación.

Situaciones especiales

- Pacientes con trasplante renal y pacientes con cirugía reconstructiva renal previa: Estas mujeres deben ser manejados en una base de caso por caso entre el nefrólogo, obstetra y el equipo de trasplante con el apoyo de Microbiología Clínica / enfermedades infecciosas consultores según sea necesario. Para los pacientes que han tenido cirugía reconstructiva renal previa es importante que ellos tienen su orina rutinariamente y son manejadas con cuidado sobre una base de caso por caso.

- $\quad$ Cálculos renales: Estas mujeres merecen especial seguimiento cercano ya que tendrán un mayor riesgo de desarrollar pielonefritis recurrente y la sepsis. Aunque no existen directrices internacionales existen dos estudios de observación que examinan opciones para el tratamiento de cálculos renales en el embarazo (Hoscan, y otros 2012). Se requiere una estrecha discusión entre nefrología y urología, obstetricia y la mujer debe gestionarse sobre una base caso por caso.

Los pacientes con bacteriemia como resultado depielonefritis

Estas mujeres pueden necesitar tratamientos prolongados con antibióticos intravenosos en función de su estado clínico y el estado del feto. conmutación temprana a los antimicrobianos de tratamiento oral puede no ser apropiado y estos casos debe ser discutido entre el equipo obstétrica y los especialistas infección local. 


\section{Tratamiento de las infecciones del tracto urinario en embarazo}

Vol. 3, núm. 2., (2019)

Stefany Gianella Campuzano Lupera; Shirley Natali Lema Sarmiento; Elvia María Córdova Cedeño; Luis Alberto Criollo Cartuche

\section{Conclusiones.}

Colectivamente, a pesar de la implementación de eficientes procedimientos de detección, las infecciones urinarias todavía se encuentran entre los trastornos más comunes relacionados con el embarazo en la gran mayoría de los países y, desafortunadamente, su incidencia no ha ido en disminución. Los síntomas pueden ser no específicos y a menudo se presentan también en mujeres embarazadas sanas. A diferencia de la población general, las infecciones asintomáticas durante el embarazo requieren tratamiento de acuerdo con la corriente recomendaciones prácticas de una serie de organismos y organizaciones internacionales. Diagnóstico precoz y tratamiento adecuado de infecciones asintomáticas permite significativamente reducir el riesgo de complicaciones maternas y fetales. La mayoría de los patógenos comunes que causan ITU demuestran sensibilidad a muchos medicamentos que pueden administrarse de manera segura durante embarazo, también en el primer trimestre. Para reducir la cantidad de complicaciones, las pruebas de uro cultivo deben ser realizado como una atención materna de rutina ya desde el I trimestre y continuar más tarde en el embarazo.

\section{Bibliografía.}

Bolívar, J. Investigación Documental. México: Pax, 2015.

Castro, J. Técnicas Documentales. México: Limusa, 2016.

Cunningham, FG, y MJ Lucas. «Urinary tract infections complicating pregnancy.» Baillieres Clinical Obstetrics and Gynaecology 8, nº 2 (1994): 353-373.

Davila, A. Concepto de terminos cientificos. Caracas: Oasis, 2015.

Etherington, I. J, y D. K. James. «Reagent strip testing of antenatal urine specimens for infection.» British Journal of Obstetrics and Gynaecology, 1993: 806-808. 


\section{Tratamiento de las infecciones del tracto urinario en embarazo}

Vol. 3, núm. 2., (2019)

Stefany Gianella Campuzano Lupera; Shirley Natali Lema Sarmiento; Elvia María Córdova Cedeño; Luis Alberto Criollo Cartuche

Guinto, V. T, B De Guia, M. R Festin, y T. Dowswell. Different antibiotic regimens for treating asymptomatic bacteriuria in pregnancy. Cd007855, Cochrane Database Systematic Review, 2010.

Hoscan, M. B, M Ekinci, A Tunckiran, T Oksay, A Ozorak, y H. Ozkardes. «Management of symptomatic ureteral calculi complicating pregnancy.» Urology, 2012: 1011-1014.

Jepson, R. G. \& Craig, J. C. Cranberries for preventing urinary tract infections. Cd001321, Cochrane Database Systematic Review, 2008.

Overturf, C. M, A. M Smith, K. A Engelbert-Fenton, A Elster, y C. J. Geiger. «Potential role of energy and nutrient intakes in decreasing the incidence of genitourinary tract infections in pregnant adolescents.» Journal of the American Dieteteic Association, 1992: 1513-1515.

Pastore, L. M, D. A Savitz, y J. Thorp. «Predictors of urinary tract infection at the first prenatal visit.» Epidemiology, 1999: 282-287.

Patterson, T. F, y V. T. Andriole. «Detection, significance, and therapy of bacteriuria in pregnancy. Update in the managed health care era.»Infectious Disease Clinics of North America, 1997: 593-608.

Perera, J. «Asymptomatic bacteriuria in pregnancy.» Sri Lanka Journal of Obstetrics and Gynaecology, 2009: 108-109.

Smaill, F. Antibiotics for asymptomatic bacteriuria in pregnancy. Cd000490., Cochrane Database Systematic Review, 2000.

Vazquez, J. C, y E. Abalos. Treatments for symptomatic urinary tract infections during pregnancy. Cd002256, Cochrane Database Systematic Review, 2011.

Widmer, M, A. M Gulmezoglu, L Mignini, y A. Roganti. Duration of treatment for asymptomatic bacteriuria during pregnancy. Cd000491, Cochrane Database Systematic Review, 2011.

$$
\text { (9) (1) } \Theta(0
$$

RECONOCIMIENTO-NOCOMERCIAL-COMPARTIRIGUAL

CC BY-NC-SA

ESTA LICENCIA PERMITE A OTROS ENTREMEZCLAR, AJUSTAR Y CONSTRUIR A PARTIR DE SU OBRA CON FINES NO

COMERCIALES, SIEMPRE Y CUANDO LE RECONOZCAN LA AUTORÍA Y SUS NUEVAS CREACIONES ESTÉN BAJO UNA LICENCIA CON LOS MISMOS TÉRMINOS. 